\title{
EFFECT OF SUGAR BEET MOLASSES AND Fe-EDHHA ON TOMATO PLANTS GROWN UNDER SALINE WATER IRRIGATION CONDITION
}

\author{
[167] \\ Fatma, K. El-Tokhy ${ }^{1}$, Tantawy ${ }^{2}$, A.S., El-Shinawy ${ }^{1}$, M.Z. and Abou-Hadid ${ }^{1}$, A.F. \\ 1- Horticulture Dept., Fac. of Agric., Ain Shams Univ., P.O. Box 68, Hadayek Shobra 11241, \\ Cairo, Egypt \\ 2- Vegetable Research Dept., National Research Center, Dokki, Giza, Egypt \\ ${ }^{*}$ Corresponding author: dr.mokhtar2000@gmail.com \\ Received 18 July, 2018 \\ Accepted 19 September, 2018
}

\section{ABSTRACT}

Salinity is a major limiting factor for tomato crop growth and productivity especially in arid and semi arid lands region. Therefore this study was conducted to study the effect of applying sugar beet molasses, priming tomato seedling in saline water and Fe-EDHHA on mitigating salinity negative effects on tomato (Lycopersicon esculentum L.) hybrid super strain B (salinity sensitive hybrid). Seedlings of tomato hybrid were transplanted on April $4^{\text {th }}$ in both seasons 2014 and 2015 in pots contains washed sandy soil, and irrigated with saline water with EC of 2000, 3000 and 4000 ppm. Beet molasses and $\mathrm{Fe}$ - EDHHA were applied at rates of 200 and $300 \mathrm{~kg} /$ fed. for beet molasses and 3 and $4 \mathrm{~kg} /$ fed. for Fe - EDHHA. Priming tomato seedlings in saline water treatment was applied at 5 and $6 \mathrm{dS} / \mathrm{m}$ for 24 hours. Data showed that all plant growth aspects such as plant height, leaf area, plant fresh and dry weights were improved under beet molasses, Fe and priming tomato seedlings in saline water treatments compared to none treated plants (control). Yield parameters also followed the same trend. Among treatments, beet molasses at a rate of $200 \mathrm{~kg} / \mathrm{fed}$. recorded the highest significant effect in mitigating salinity negative effects. It could be concluded that beet molasses, priming tomato seedling in saline water and $\mathrm{Fe}$ - EDHHA treatments were more effective and efficient in mitigating salinity stress on tomato plants.

Keywords: Tomato, Salinity, Beet molasses, Fe EDHHA, and Total Yield.

\section{INTRODUCTION}

Worldwide, more than 45 million hectares of irrigated land have been damaged by salt and 1.5 million hectares are taken out of production each year as a result of high salinity levels in the soil (Munns and Tester, 2008). Salinity problem has been reported to affect about $45 \%$ of the total Egyptian area (including $26 \%$ of water bodies) according to Gehad, (2003). Since that report, no further reports have been found about the development of that problem in Egypt although its spreading is noticeable through personal communications with Egyptian growers. The negative effects of salinity have been reported on growth and production of many crops such as mungbean (Salama, 2003), green bean (Abdel-Mawgoud et al 2010) and Gomaa, (2017); tomato (Salama et al (2012) and Sweet pepper (Abdel-Mawgoud et al 2004). For these reasons different attempts have been tried in order to alleviate the negative effects of salinity on plant performance. For instance, trails for manipulating greenhouse climate (Li, (2000); Abdel-Mawgoud et al (2004), application of amino acids and growth regulators (Tantawy et al 2009a) and using of environmental friendly materials (Abdel-Mawgoud et al (2010) and Tantawy et al (2009b) as well as using physical treatments such as hardening (Shafshak et al (2008) and Tantawy et al (2009c) and some mineral fertilization (Salama et al 2012). Salama et al (2012) ameliorated the negative effects of salinity by using Zn-EDTA. Other micro nutrients may have similar ameliorating effects on some vegetable crops such as tomato. molasses on tomato plant growth and productivity under saline 
water irrigation attributed to molasses contain glycinebetaine material is as a compatible solute in osmotic adjustment of the cytoplasmic compartments where it may accumulate while ions are sequestered in the vacuole Salisbury and Ross, (1992). Tantawy, (2007) found that beet molasses contained Water $17 \%$,Sucrose 66\%, Fructose 1\%, Glucose $1 \%$,Glycinebetaine 6\%,Amino acids 8\% ,Sterols $0.3 \%$, Phospholipids $0.5 \%$, and Wax $0.2 \%$. Tomato is a major vegetable crop in Egypt ranking the first crop in terms of value (2995.412 million dollars) and the third in terms of quantity of production (8105260 metric tons) FAOSTAT, (2011). According to FAOSTAT (2011), Egypt is ranked the fifth on the world in terms of production and value of tomato crop with a total harvested area reached 212446 ha. The cultivation of tomato crop is very favorable by the growers because of its moderate tolerance to salinity which enable the plant to improve its performance by application of some agricultural practices specially those treatments that provide nutrients to the plants. Therefore, the aim of this study is to investigate the ameliorating effects of $\mathrm{Fe}$, beet molasses and priming tomato seedling in saline water on salinity impacts on tomato crop.

\section{MATERIALS AND METHODS}

Two pot experiments were carried out during two successive summer seasons of 2013 and 2014 in a private farm in Abou Ghalib region, Giza governorate to investigate the effects of using beet molasses at rates of 200 and $300 \mathrm{~kg} / \mathrm{fed}$. and Fe - EDHHA 3 and $4 \mathrm{~kg} \mathrm{/} \mathrm{fed.} \mathrm{and} \mathrm{priming} \mathrm{tomato} \mathrm{seedlings} \mathrm{in}$ saline water treatment at 5 and $6 \mathrm{dS} / \mathrm{m}$ for 24 hours to alleviate the effect of different salinity levels on the growth and yield of tomato plants hybrid super strain B under soil sandy. Seeds of tomato were sown on $11^{\text {th }}$ February in foam trays under the condition of greenhouse in both seasons of both studies. The seedlings of tomato hybrids were transplanted on April $4^{\text {th }}$ in both seasons at the stage of $5^{\text {th }}$ true leaf in plastic pots. The pots size was 30 $\mathrm{cm}$ in diameter and $50 \mathrm{~cm}$ depth. The pots were filled with $13 \mathrm{~kg}$ washed sand. Plants were irrigated by three concentrations of saline water, 2000, 3000 and 4000 ppm which were obtained by mixing water from Karoun Lake with the tap water (260 ppm).
The plastic pots were perforated to allow drainage. Two uniform seedlings were transplanted in each pot, and placed in open field. Thinning took place after 15 days from transplanting leaving one plant only in each pot. Plants were hand irrigated with water for two weeks until plant establishments was assured in the growing media then salinity water was applied periodically every 2-3 days with 1000 $\mathrm{ml} / \mathrm{pot}$ to keep the water content at field capacity.

The experiment included 315 pots resulting from combination of 21 treatments with 3 replicates and every replicate consisted of 5 pots. Where, tomato seedlings were supplied by an aqueous solution of beet molasses and lor Fe-EDHHA three times during the growing period, i.e. after 3,6 and 9 weeks from transplanting.

\section{Data Recorded}

Plant height $(\mathrm{cm})$, plant fresh and dry weight $(\mathrm{g})$ and leaf number were recorded on plants at the end of the seasons. Meanwhile leaf area was recorded in the fourth leaf from the apex. Total fruits yield as $\mathrm{g}$ / plant was determined as total weight of fruits during the harvest period, marketable fruits yield (g/plant) was determined after excluding unmarketable fruits. (g/plant). Chlorophyll content was measured on the forth leaf from the apex using Minolta Chlorophyll meter Model 501, Yadava, (1986). Mineral content was analyzed in the fourth leaf from the apex where samples were dried in an oven at $70{ }^{\circ} \mathrm{C}$ until a constant mass was reached and then they were grounded for chemical analysis. Total nitrogen was determined using the microKjeldahl method Kacar, (1972). Phosphorus was determined spectrophotometrically according to Troug and Meyer (1939). Potassium content was determined using an atomic absorption spectrophotometer (Brown and Lilleland, 1946). Fe was determined using atomic-absorption as described by Chapman and Pratt (1982). Sodium analyzed and measured in leaves by flame-photometer set as described by Brown and Lilleland (1946). Total proline content was measured according to Bates et al (1973).

The experimental treatments were arranged by applying the split plot design with three replicates where, salinity levels treatments were arranged in the main plots and beet molasses, Fe - EDHHA and priming tomato seedlings in saline water treatments were arranged in the sub plots. The 
obtained data were statistically analyzed by the method described by Gomez and Gomez (1984).

\section{RESULTS}

Table (1) show the effect of the salinity levels, organic and chemical compounds and the interactions among them on plant height, fresh and dry weight respectively. As salinity level increased, there was a significant negative effect on plant height, fresh and dry weight. These trends were consistent in both growing seasons of the experiment. Meanwhile, the application of organic and chemical compounds improved plant performance compared to control. Molasses at rate of $200 \mathrm{~kg}$ /fed. recorded the highest positive effects. Howev$\mathrm{er}$, in all salinity levels, molasses at rate of $200 \mathrm{~kg}$ /fed. showed superiority in the positive effect compared to other treatments and this difference was significant. The interaction effect showed that the high values of plant height, fresh and dry weight were noticed with that plants which irrigated by water contained $2000 \mathrm{ppm}$ and supplied molasses at $200 \mathrm{~kg} / \mathrm{fed}$. On the opposite, occurred when using irrigation water contain 4000 with no supplied any organic and chemical materials, in $1^{\text {st }}$ and $2^{\text {nd }}$ seasons.

Table (2) demonstrates the effects of the applied treatments on total chlorophyll content and leaf area of the tomato plants and the interaction effects on the same parameters. It is obvious from the recorded data that there is a negative effect of salinity on total chlorophyll content and leaf area which was significant compared to control. Although the application of all treatments in this study reduced that negative effect of salinity, there was clear superiority pattern of molasses at rate of 200 $\mathrm{kg} /$ fed compared to other treatments. The interaction effect showed that the best values of total chlorophyll content and leaf area were noticed with the plants which irrigated by water contained 2000 ppm and supplied molasses at $200 \mathrm{~kg} / \mathrm{fed}$. On the opposite, occurred when using irrigation water contain 4000 ppm with no supplied any organic and chemical materials, in $1^{\text {st }}$ and $2^{\text {nd }}$ seasons.

The observed pattern in the vegetative growth was also recorded in the yield and its components. Total yield was significantly reduced as salinity level increased (Table 3). Meanwhile organic and chemical compounds application significantly mitigated those negative effects and total yield was markedly improved. Among organic and chemical compounds applications, molasses at rate of 200 $\mathrm{kg} / \mathrm{fed}$ showed the highest mitigation effect on total yield under all salinity levels. Similarly, marketable yield was severely reduced as salinity level increased significantly. The application of organic and chemical compounds improved the fraction of marketable yield and reduced the negative effects of salinity significantly.

Molasses at rate of $200 \mathrm{~kg} / \mathrm{fed}$ recorded the highest effect in improving marketable yield compared to all other treatments. On the contrary of the all above results, only unmarketable yield is the parameter which showed an opposite trend where salinity increment increased the fraction of unmarketable yield. The application of organic and chemical compounds reduced that parameter with molasses at rate of $200 \mathrm{~kg} / \mathrm{fed}$ which recorded the highest effects.

Similarly, nutrients such as N, P and K \% showed a reduction as salinity level increased (Table 4), these reductions were mitigated by the application of organic and chemical compounds with molasses at rate of $200 \mathrm{~kg} / \mathrm{fed}$ which recorded the highest positive effect. All organic and chemical compounds applications effects were significantly higher compared to control under each salinity level. Nevertheless, salinity levels remained the dominant factor in its effect. As it was expected, $\mathrm{Na} \%$ increase in plant tissues as salinity level increased (Table 5). Meanwhile the application of organic and chemical compounds significantly reduced $\mathrm{Na} \%$ and the lowest \% recorded under the treatment of molasses at rate of $200 \mathrm{~kg}$ /fed. The interaction of the treatments shows clearly the dominant effect of salinity on all the treatments.

Proline content as a parameter of stress showed a strong positive response to the increment in salinity level. That response was significantly reduced by the application of organic and chemical compounds (Table 5). The obtained data cleared that, the plants which irrigated with the highest salinity level, i.e. $4000 \mathrm{ppm}$ resulted the highest proline percentage. Generally speaking, increasing the salinity levels significantly increased the percentage of proline in leaves tissue in both seasons of study, whereas, the lowest value of proline was produced by using the lowest salinity level, i.e. 2000 ppm. Within different organic and chemical compounds, control treatment recorded the highest proline percentage. On the contrary the lowest proline value was associated with that plants treated with molasses at $200 \mathrm{~kg} / \mathrm{fed}$. Under different salinity levels, supplying the organic and chemical compounds caused a significant reduction in proline percentage compared with control 
Table 1. Effect of different salinity levels, organic and chemical compounds on plant height, fresh and dry weight of tomato plants in 2014 and 2015 seasons

\begin{tabular}{|c|c|c|c|c|c|c|c|}
\hline \multirow{2}{*}{$\begin{array}{l}\text { Salinity } \\
\text { levels }\end{array}$} & \multirow{2}{*}{$\begin{array}{l}\text { Organic and } \\
\text { Chemical } \\
\text { Compound }\end{array}$} & \multicolumn{2}{|c|}{ Plant height (cm) } & \multicolumn{2}{|c|}{$\begin{array}{c}\text { Plant fresh weight } \\
\text { (g / plant) }\end{array}$} & \multicolumn{2}{|c|}{$\begin{array}{c}\text { Plant dry weight } \\
\text { (g / plant) }\end{array}$} \\
\hline & & $\begin{array}{c}2014 \\
\text { season }\end{array}$ & $\begin{array}{c}2015 \\
\text { season }\end{array}$ & $\begin{array}{c}2014 \\
\text { season }\end{array}$ & $\begin{array}{c}2015 \\
\text { season }\end{array}$ & $\begin{array}{c}2014 \\
\text { season }\end{array}$ & $\begin{array}{c}2015 \\
\text { season }\end{array}$ \\
\hline \multirow{7}{*}{2000 ppm } & Control & 43.84 & 42.28 & 74.54 & 76.26 & 26.60 & 27.76 \\
\hline & Fe- $3 \mathrm{~kg} / \mathrm{fed}$ & 54.31 & 52.58 & 103.86 & 105.27 & 28.81 & 28.77 \\
\hline & Fe- $4 \mathrm{~kg} / \mathrm{fed}$ & 54.43 & 52.00 & 113.13 & 113.92 & 28.00 & 29.36 \\
\hline & Molasses $200 \mathrm{~kg} / \mathrm{fed}$ & 58.10 & 57.52 & 158.96 & 155.60 & 30.15 & 30.76 \\
\hline & Molasses $300 \mathrm{~kg} / \mathrm{fed}$ & 57.41 & 55.38 & 120.85 & 124.00 & 29.42 & 30.21 \\
\hline & $\begin{array}{c}\text { Priming at } 5 \text { EC / } \\
24 \mathrm{~h}\end{array}$ & 52.29 & 50.48 & 99.88 & 115.43 & 27.69 & 28.86 \\
\hline & $\begin{array}{c}\text { Priming at } 6 \text { EC / } \\
24 \mathrm{~h}\end{array}$ & 51.28 & 49.02 & 98.16 & 101.73 & 27.63 & 28.26 \\
\hline Mean & & 53.09 & 51.32 & 109.91 & 113.17 & 28.33 & 29.14 \\
\hline \multirow{7}{*}{3000 ppm } & Control & 36.92 & 34.77 & 51.44 & 51.94 & 31.34 & 30.09 \\
\hline & Fe- $3 \mathrm{~kg} / \mathrm{fed}$ & 49.93 & 47.96 & 84.65 & 95.52 & 32.83 & 32.18 \\
\hline & Fe- $4 \mathrm{~kg} / \mathrm{fed}$ & 52.23 & 50.19 & 96.56 & 100.83 & 33.47 & 32.36 \\
\hline & Molasses $200 \mathrm{~kg} / \mathrm{fed}$ & 54.76 & 52.83 & 130.65 & 134.08 & 33.93 & 33.54 \\
\hline & Molasses $300 \mathrm{~kg} / \mathrm{fed}$ & 53.53 & 52.11 & 108.95 & 112.38 & 33.80 & 33.07 \\
\hline & $\begin{array}{c}\text { Priming at } 5 \text { EC / } \\
24 \mathrm{~h}\end{array}$ & 51.21 & 49.06 & 96.76 & 92.11 & 35.12 & 31.79 \\
\hline & $\begin{array}{c}\text { Priming at } 6 \mathrm{EC} / \\
24 \mathrm{~h}\end{array}$ & 49.91 & 48.03 & 88.84 & 95.54 & 34.72 & 31.66 \\
\hline Mean & & 49.79 & 47.85 & 93.98 & 97.49 & 33.60 & 32.10 \\
\hline \multirow{7}{*}{4000 ppm } & Control & 34.36 & 32.12 & 39.13 & 43.50 & 32.60 & 32.60 \\
\hline & Fe- $3 \mathrm{~kg} / \mathrm{fed}$ & 48.98 & 45.37 & 77.14 & 81.42 & 33.75 & 33.57 \\
\hline & $\mathrm{Fe}-\mathbf{4 k g} / \mathrm{fed}$ & 50.01 & 46.29 & 86.81 & 89.42 & 34.02 & 34.20 \\
\hline & Molasses $200 \mathrm{~kg} / \mathrm{fed}$ & 52.39 & 48.71 & 103.32 & 113.80 & 37.03 & 35.01 \\
\hline & Molasses $300 \mathrm{~kg} / \mathrm{fed}$ & 51.14 & 47.76 & 94.07 & 97.94 & 35.96 & 33.87 \\
\hline & $\begin{array}{c}\text { Priming at } 5 \text { EC / } \\
24 \mathrm{~h}\end{array}$ & 48.56 & 44.74 & 85.94 & 85.60 & 33.92 & 32.87 \\
\hline & $\begin{array}{c}\text { Priming at } 6 \text { EC / } \\
24 \mathrm{~h}\end{array}$ & 46.48 & 43.35 & 84.86 & 78.67 & 33.65 & 32.55 \\
\hline Mean & & 47.42 & 44.05 & 81.61 & 84.34 & 34.42 & 33.52 \\
\hline \multirow{7}{*}{$\begin{array}{l}\text { Mean of } \\
\text { Organic and } \\
\text { chemical } \\
\text { compounds }\end{array}$} & Control & 38.37 & 36.39 & 55.04 & 57.23 & 30.18 & 30.15 \\
\hline & Fe- $3 k g$ / fed. & 51.07 & 48.64 & 94.20 & 97.71 & 31.80 & 31.51 \\
\hline & Fe- $4 \mathrm{~kg} / \mathrm{fed}$ & 52.22 & 49.49 & 98.83 & 101.39 & 31.83 & 31.97 \\
\hline & Molasses $200 \mathrm{~kg} / \mathrm{fed}$ & 55.08 & 53.02 & 130.98 & 134.49 & 33.70 & 33.10 \\
\hline & Molasses $300 \mathrm{~kg} / \mathrm{fed}$ & 54.03 & 51.75 & 107.96 & 111.44 & 33.06 & 32.38 \\
\hline & $\begin{array}{c}\text { Priming at } 5 \text { EC / } \\
24 \mathrm{~h}\end{array}$ & 50.69 & 48.09 & 90.60 & 94.07 & 32.25 & 31.17 \\
\hline & $\begin{array}{c}\text { Priming at } 6 \text { EC / } \\
24 \mathrm{~h}\end{array}$ & 49.22 & 46.80 & 88.55 & 91.98 & 32.00 & 30.83 \\
\hline \multirow{3}{*}{ L.S.D. at $5 \%$} & $\begin{array}{c}\text { Salinity } \\
\text { Organic and }\end{array}$ & 1.02 & 0.92 & 0.43 & 0.35 & 0.21 & N.S. \\
\hline & $\begin{array}{l}\text { Chemical } \\
\text { compounds }\end{array}$ & 0.29 & 0.34 & 0.55 & 0.46 & 0.05 & 0.04 \\
\hline & Interaction & 0.51 & 0.58 & 0.95 & 0.80 & 0.08 & 0.07 \\
\hline
\end{tabular}


Table 2. Effect of different salinity levels, organic and chemical compounds on leaf area and total chlorophyll in tomato plants in 2014 and 2015 seasons

\begin{tabular}{|c|c|c|c|c|c|}
\hline \multirow{2}{*}{ Salinity levels } & \multirow{2}{*}{$\begin{array}{l}\text { Organic and Chemical } \\
\text { Compound }\end{array}$} & \multicolumn{2}{|c|}{$\begin{array}{l}\text { Leaf area } \\
\qquad\left(\mathrm{cm}^{2}\right)\end{array}$} & \multicolumn{2}{|c|}{$\begin{array}{c}\text { Total } \\
\text { Chlorophyll } \\
\text { (SPAD) } \\
\end{array}$} \\
\hline & & $\begin{array}{c}2014 \\
\text { season }\end{array}$ & $\begin{array}{c}2015 \\
\text { season }\end{array}$ & $\begin{array}{c}2014 \\
\text { season }\end{array}$ & $\begin{array}{l}2015 \\
\text { season }\end{array}$ \\
\hline \multirow{7}{*}{2000 ppm } & Control & 68.61 & 74.17 & 48.84 & 49.59 \\
\hline & Fe- 3kg / fed. & 77.17 & 82.76 & 57.25 & 58.84 \\
\hline & Fe- 4kg /fed. & 77.32 & 82.03 & 58.08 & 61.42 \\
\hline & Molasses 200 kg/fed & 80.26 & 85.03 & 62.11 & 64.21 \\
\hline & Molasses $300 \mathrm{~kg} / \mathrm{fed}$ & 78.67 & 83.97 & 60.26 & 62.84 \\
\hline & Priming at $5 \mathrm{EC} / 24 \mathrm{~h}$ & 75.91 & 82.30 & 56.44 & 58.67 \\
\hline & Priming at $6 \mathrm{EC} / 24 \mathrm{~h}$ & 75.01 & 81.17 & 55.65 & 57.05 \\
\hline Mean & & 76.13 & 81.63 & 56.95 & 58.95 \\
\hline \multirow{7}{*}{3000 ppm } & Control & 62.27 & 63.48 & 42.35 & 42.89 \\
\hline & Fe- $3 \mathrm{~kg} /$ fed. & 69.95 & 70.62 & 52.57 & 52.85 \\
\hline & Fe- $4 k g$ /fed. & 70.82 & 72.20 & 53.73 & 54.05 \\
\hline & Molasses $200 \mathrm{~kg} / \mathrm{fed}$ & 74.73 & 75.08 & 56.27 & 57.25 \\
\hline & Molasses $300 \mathrm{~kg} / \mathrm{fed}$ & 72.92 & 73.85 & 55.45 & 55.37 \\
\hline & Priming at $5 \mathrm{EC} / 24 \mathrm{~h}$ & 69.36 & 70.45 & 51.59 & 52.51 \\
\hline & Priming at $6 \mathrm{EC} / 24 \mathrm{~h}$ & 68.37 & 69.53 & 51.13 & 52.20 \\
\hline Mean & & 69.77 & 70.75 & 51.87 & 52.45 \\
\hline \multirow{7}{*}{4000 ppm } & Control & 69.77 & 58.12 & 33.47 & 34.78 \\
\hline & Fe- $3 \mathrm{~kg} /$ fed. & 57.18 & 66.87 & 48.91 & 50.40 \\
\hline & Fe- $4 k g$ /fed. & 65.96 & 67.15 & 50.09 & 50.59 \\
\hline & Molasses 200 kg/fed & 66.70 & 68.98 & 51.71 & 52.40 \\
\hline & Molasses $300 \mathrm{~kg} / \mathrm{fed}$ & 66.38 & 67.87 & 51.39 & 52.08 \\
\hline & Priming at $5 \mathrm{EC} / 24 \mathrm{~h}$ & 66.26 & 66.69 & 48.85 & 48.56 \\
\hline & Priming at $6 \mathrm{EC} / 24 \mathrm{~h}$ & 65.14 & 66.39 & 48.37 & 47.55 \\
\hline Mean & & 65.06 & 66.01 & 47.54 & 48.05 \\
\hline \multirow{7}{*}{$\begin{array}{l}\text { Mean of } \\
\text { Organic and chemical } \\
\text { compounds }\end{array}$} & Control & 62.69 & 65.26 & 41.55 & 42.42 \\
\hline & Fe- $3 k g$ / fed. & 71.03 & 73.42 & 52.91 & 54.03 \\
\hline & Fe- $4 \mathrm{~kg} / \mathrm{fed}$ & 71.51 & 73.79 & 53.97 & 55.35 \\
\hline & Molasses $200 \mathrm{~kg} / \mathrm{fed}$ & 74.27 & 76.36 & 56.70 & 57.95 \\
\hline & Molasses $300 \mathrm{~kg} / \mathrm{fed}$ & 72.76 & 75.23 & 55.70 & 56.76 \\
\hline & Priming at $5 \mathrm{EC} / 24 \mathrm{~h}$ & 70.51 & 73.15 & 52.29 & 53.25 \\
\hline & Priming at $6 \mathrm{EC} / 24 \mathrm{~h}$ & 69.51 & 72.36 & 51.72 & 52.27 \\
\hline \multirow{3}{*}{ L.S.D. at $5 \%$} & Salinity & 0.79 & 0.51 & 2.64 & 2.81 \\
\hline & $\begin{array}{c}\text { Organic and Chemical } \\
\text { Compound }\end{array}$ & 0.13 & 0.36 & 0.13 & 0.19 \\
\hline & Interaction & 0.22 & 0.62 & 0.22 & 0.33 \\
\hline
\end{tabular}


Table 3. Effect of different salinity levels, organic and chemical compounds on total yield, marketable yield and unmarketable yield of tomato plants in 2014 and 2015 seasons

\begin{tabular}{|c|c|c|c|c|c|c|c|}
\hline \multirow{2}{*}{ Salinity levels } & \multirow{2}{*}{$\begin{array}{l}\text { Organic and Chemical } \\
\text { Compound }\end{array}$} & \multicolumn{2}{|c|}{$\begin{array}{l}\text { Total yield } \\
\text { (g/ plant) }\end{array}$} & \multicolumn{2}{|c|}{$\begin{array}{l}\text { Marketable yield } \\
\text { (g/ plant) }\end{array}$} & \multicolumn{2}{|c|}{$\begin{array}{c}\text { Unmarketable } \\
\text { yield } \\
\text { (g/ plant) }\end{array}$} \\
\hline & & $\begin{array}{c}2014 \\
\text { season }\end{array}$ & $\begin{array}{c}2015 \\
\text { season }\end{array}$ & $\begin{array}{c}2014 \\
\text { season }\end{array}$ & $\begin{array}{c}2015 \\
\text { season }\end{array}$ & $\begin{array}{c}2014 \\
\text { season }\end{array}$ & $\begin{array}{c}2015 \\
\text { season }\end{array}$ \\
\hline \multirow{7}{*}{2000 ppm } & Control & 567.68 & 569.98 & 468.75 & 472.56 & 98.93 & 97.42 \\
\hline & Fe- $3 k g$ / fed. & 641.22 & 636.45 & 556.70 & 560.02 & 88.00 & 76.44 \\
\hline & Fe- 4 kg /fed. & 644.70 & 644.59 & 561.72 & 569.27 & 77.37 & 87.73 \\
\hline & Molasses $200 \mathrm{~kg} / \mathrm{fed}$ & 651.84 & 657.63 & 576.54 & 586.72 & 75.30 & 70.92 \\
\hline & Molasses $300 \mathrm{~kg} / \mathrm{fed}$ & 645.42 & 656.99 & 568.74 & 571.73 & 76.68 & 72.86 \\
\hline & Priming at $5 \mathrm{EC} / 24 \mathrm{~h}$ & 639.09 & 637.01 & 554.00 & 551.51 & 87.21 & 85.49 \\
\hline & Priming at $6 \mathrm{EC} / 24 \mathrm{~h}$ & 627.65 & 626.95 & 538.20 & 543.88 & 89.45 & 83.07 \\
\hline Mean & & 631.09 & 632.80 & 546.38 & 550.81 & 84.71 & 81.99 \\
\hline \multirow{7}{*}{3000 ppm } & Control & 514.07 & 518.76 & 394.48 & 410.07 & 119.59 & 108.70 \\
\hline & Fe- $3 \mathrm{~kg} / \mathrm{fed}$ & 545.15 & 582.49 & 451.69 & 493.36 & 97.63 & 97.51 \\
\hline & $\mathrm{Fe}-4 \mathrm{~kg} / \mathrm{fed}$. & 548.77 & 590.04 & 457.89 & 488.88 & 87.25 & 89.12 \\
\hline & Molasses $200 \mathrm{~kg} / \mathrm{fed}$ & 560.43 & 597.00 & 479.21 & 514.55 & 81.22 & 82.45 \\
\hline & Molasses $300 \mathrm{~kg} / \mathrm{fed}$ & 551.26 & 591.36 & 465.80 & 505.40 & 82.97 & 85.96 \\
\hline & Priming at $5 \mathrm{EC} / 24 \mathrm{~h}$ & 541.01 & 580.86 & 443.39 & 483.35 & 98.91 & 98.44 \\
\hline & Priming at $6 \mathrm{EC} / 24 \mathrm{~h}$ & 533.51 & 572.35 & 434.60 & 473.91 & 99.56 & 101.16 \\
\hline Mean & & 542.03 & 576.12 & 446.72 & 481.36 & 95.31 & 94.76 \\
\hline \multirow{7}{*}{4000 ppm } & Control & 455.25 & 450.56 & 314.24 & 311.85 & 142.34 & 138.71 \\
\hline & Fe- $3 \mathrm{~kg} / \mathrm{fed}$ & 498.04 & 511.65 & 385.76 & 390.81 & 113.91 & 109.58 \\
\hline & Fe- 4 kg /fed. & 500.21 & 514.98 & 397.57 & 414.17 & 102.10 & 100.81 \\
\hline & Molasses $200 \mathrm{~kg} / \mathrm{fed}$ & 524.96 & 535.29 & 424.99 & 435.92 & 101.31 & 99.37 \\
\hline & Molasses $300 \mathrm{~kg} / \mathrm{fed}$ & 511.26 & 516.75 & 410.49 & 416.46 & 101.81 & 100.28 \\
\hline & Priming at $5 \mathrm{EC} / 24 \mathrm{~h}$ & 489.68 & 501.08 & 377.10 & 378.97 & 115.78 & 110.27 \\
\hline & Priming at $6 \mathrm{EC} / 24 \mathrm{~h}$ & 485.35 & 488.55 & 368.14 & 396.36 & 118.54 & 115.29 \\
\hline Mean & & 494.96 & 502.69 & 382.61 & 392.08 & 113.68 & 110.62 \\
\hline \multirow{7}{*}{$\begin{array}{l}\text { Mean of } \\
\text { Organic and } \\
\text { chemical } \\
\text { compounds }\end{array}$} & Control & 512.33 & 513.10 & 392.49 & 398.16 & 120.29 & 114.94 \\
\hline & Fe- $3 \mathrm{~kg} / \mathrm{fed}$ & 560.76 & 573.34 & 464.72 & 481.40 & 99.58 & 96.57 \\
\hline & $\mathrm{Fe}-4 \mathrm{~kg} / \mathrm{fed}$ & 565.39 & 584.23 & 472.39 & 490.77 & 88.81 & 91.94 \\
\hline & Molasses 200 kg/fed & 579.08 & 596.64 & 493.58 & 512.40 & 85.94 & 84.24 \\
\hline & Molasses 300 kg/fed & 568.48 & 587.34 & 481.67 & 497.86 & 87.25 & 86.37 \\
\hline & Priming at $5 \mathrm{EC} / 24 \mathrm{~h}$ & 557.30 & 570.31 & 458.16 & 471.28 & 101.12 & 97.53 \\
\hline & Priming at $6 \mathrm{EC} / 24 \mathrm{~h}$ & 548.84 & 568.81 & 446.98 & 471.38 & 102.30 & 98.93 \\
\hline \multirow{3}{*}{ L.S.D. at $5 \%$} & Salinity & 8.57 & 6.34 & 8.03 & 7.74 & 1.43 & 1.39 \\
\hline & $\begin{array}{c}\text { Organic and Chemical } \\
\text { Compound }\end{array}$ & 0.61 & 0.75 & 0.89 & 1.00 & 0.33 & 0.29 \\
\hline & Interaction & 1.05 & 1.29 & 1.55 & 1.72 & 0.57 & 0.51 \\
\hline
\end{tabular}


Table 4. Effect of different salinity levels, organic and chemical compounds on nitrogen, phosphorus and potassium percentages (\%) of tomato leaves in 2014 and 2015 seasons

\begin{tabular}{|c|c|c|c|c|c|c|c|}
\hline \multirow{2}{*}{ Salinity levels } & \multirow{2}{*}{$\begin{array}{c}\text { Organic and Chemical } \\
\text { Compound }\end{array}$} & \multicolumn{2}{|c|}{$\begin{array}{c}N \\
(\%)\end{array}$} & \multicolumn{2}{|c|}{$\begin{array}{c}P \\
(\%)\end{array}$} & \multicolumn{2}{|c|}{$\begin{array}{c}\mathrm{K} \\
(\%)\end{array}$} \\
\hline & & $\begin{array}{c}2014 \\
\text { season }\end{array}$ & $\begin{array}{c}2015 \\
\text { season }\end{array}$ & $\begin{array}{c}2014 \\
\text { season }\end{array}$ & $\begin{array}{c}2015 \\
\text { season }\end{array}$ & $\begin{array}{c}2014 \\
\text { season }\end{array}$ & $\begin{array}{c}2015 \\
\text { season }\end{array}$ \\
\hline \multirow{7}{*}{2000 ppm } & Control & 3.08 & 2.85 & 0.44 & 0.41 & 2.21 & 2.31 \\
\hline & Fe- $3 \mathrm{~kg} /$ fed. & 3.39 & 3.19 & 0.50 & 0.48 & 2.90 & 2.99 \\
\hline & Fe- 4 kg /fed. & 3.55 & 3.39 & 0.51 & 0.50 & 2.97 & 3.11 \\
\hline & Molasses 200 kg/fed & 3.85 & 3.62 & 0.53 & 0.51 & 3.49 & 3.49 \\
\hline & Molasses 300 kg/fed & 3.80 & 3.56 & 0.52 & 0.50 & 3.16 & 3.28 \\
\hline & Priming at $5 \mathrm{EC} / 24 \mathrm{~h}$ & 3.39 & 3.03 & 0.51 & 0.50 & 2.82 & 2.93 \\
\hline & Priming at $6 \mathrm{EC} / 24 \mathrm{~h}$ & 3.28 & 2.96 & 0.48 & 0.48 & 2.50 & 2.82 \\
\hline \multicolumn{2}{|r|}{ Mean } & 3.48 & 3.23 & 0.50 & 0.49 & 2.86 & 2.99 \\
\hline \multirow{7}{*}{3000 ppm } & Control & 2.29 & 2.01 & 0.38 & 0.32 & 1.90 & 2.06 \\
\hline & Fe- 3kg / fed. & 2.83 & 2.70 & 0.48 & 0.45 & 2.70 & 2.82 \\
\hline & Fe- $4 \mathrm{~kg} / \mathrm{fed}$ & 2.89 & 2.75 & 0.48 & 0.45 & 2.75 & 2.67 \\
\hline & Molasses 200 kg/fed & 3.13 & 3.06 & 0.50 & 0.48 & 3.22 & 3.18 \\
\hline & Molasses 300 kg/fed & 3.11 & 2.98 & 0.49 & 0.46 & 3.03 & 2.97 \\
\hline & Priming at $5 \mathrm{EC} / 24 \mathrm{~h}$ & 2.79 & 2.56 & 0.47 & 0.45 & 2.68 & 2.76 \\
\hline & Priming at $6 \mathrm{EC} / 24 \mathrm{~h}$ & 2.60 & 2.53 & 0.45 & 0.43 & 2.31 & 2.59 \\
\hline \multicolumn{2}{|r|}{ Mean } & 2.81 & 2.66 & 0.46 & 0.44 & 2.65 & 2.72 \\
\hline \multirow{7}{*}{4000 ppm } & Control & 1.95 & 1.46 & 0.30 & 0.28 & 1.64 & 1.92 \\
\hline & Fe- $3 \mathrm{~kg} /$ fed. & 2.52 & 2.03 & 0.39 & 0.37 & 2.31 & 2.41 \\
\hline & $\mathrm{Fe}-4 \mathrm{~kg} / \mathrm{fed}$ & 2.55 & 2.13 & 0.40 & 0.37 & 2.45 & 2.42 \\
\hline & Molasses 200 kg/fed & 2.75 & 2.36 & 0.42 & 0.39 & 2.80 & 2.73 \\
\hline & Molasses 300 kg/fed & 2.72 & 2.29 & 0.41 & 0.38 & 2.61 & 2.50 \\
\hline & Priming at $5 \mathrm{EC} / 24 \mathrm{~h}$ & 2.46 & 2.01 & 0.38 & 0.37 & 2.29 & 2.34 \\
\hline & Priming at $6 \mathrm{EC} / 24 \mathrm{~h}$ & 2.40 & 1.82 & 0.37 & 0.35 & 1.97 & 2.15 \\
\hline \multicolumn{2}{|r|}{ Mean } & 2.48 & 2.01 & 0.38 & 0.36 & 2.29 & 2.35 \\
\hline \multirow{7}{*}{$\begin{array}{c}\text { Mean of } \\
\text { Organic and } \\
\text { chemical com- } \\
\text { pounds }\end{array}$} & Control & 2.44 & 2.11 & 0.37 & 0.34 & 1.92 & 2.10 \\
\hline & Fe- $3 \mathrm{~kg} / \mathrm{fed}$ & 2.91 & 2.64 & 0.45 & 0.44 & 2.64 & 2.74 \\
\hline & $\mathrm{Fe}-4 \mathrm{~kg} / \mathrm{fed}$ & 3.00 & 2.76 & 0.46 & 0.44 & 2.72 & 2.73 \\
\hline & Molasses 200 kg/fed & 3.24 & 3.01 & 0.48 & 0.46 & 3.17 & 3.14 \\
\hline & Molasses 300 kg/fed & 3.21 & 2.94 & 0.47 & 0.45 & 2.93 & 2.91 \\
\hline & Priming at $5 \mathrm{EC} / 24 \mathrm{~h}$ & 2.88 & 2.53 & 0.45 & 0.44 & 2.59 & 2.68 \\
\hline & Priming at $6 \mathrm{EC} / 24 \mathrm{~h}$ & 2.76 & 2.43 & 0.43 & 0.42 & 2.26 & 2.52 \\
\hline \multirow{3}{*}{ L.S.D. at $5 \%$} & Salinity & 0.53 & 0.32 & 0.04 & 0.05 & N.S. & N.S. \\
\hline & $\begin{array}{c}\text { Organic and Chemical } \\
\text { Compound }\end{array}$ & 0.03 & 0.04 & 0.01 & 0.01 & 0.28 & 0.22 \\
\hline & Interaction & 0.06 & 0.07 & N.S. & 0.02 & N.S. & N.S. \\
\hline
\end{tabular}


Table 5. Effect of different salinity levels, organic and chemical compounds on iron (ppm), Sodium and proline percentages (\%) of tomato leaves in 2014 and 2015 seasons

\begin{tabular}{|c|c|c|c|c|c|c|c|}
\hline \multirow{2}{*}{ Salinity levels } & \multirow{2}{*}{$\begin{array}{c}\text { Organic and Chemical } \\
\text { Compound }\end{array}$} & \multicolumn{2}{|c|}{$\begin{array}{c}\mathrm{Fe} \\
(\mathrm{ppm})\end{array}$} & \multicolumn{2}{|c|}{$\begin{array}{l}\mathrm{Na} \\
(\%)\end{array}$} & \multicolumn{2}{|c|}{$\begin{array}{l}\text { Proline } \\
(\%)\end{array}$} \\
\hline & & $\begin{array}{l}2014 \\
\text { season }\end{array}$ & $\begin{array}{c}2015 \\
\text { season }\end{array}$ & $\begin{array}{l}2014 \\
\text { season }\end{array}$ & $\begin{array}{l}2015 \\
\text { season }\end{array}$ & $\begin{array}{l}2014 \\
\text { season }\end{array}$ & $\begin{array}{l}2015 \\
\text { season }\end{array}$ \\
\hline \multirow{7}{*}{2000 ppm } & Control & 52.00 & 56.11 & 0.70 & 0.75 & 0.063 & 0.066 \\
\hline & Fe- 3kg / fed. & 64.12 & 71.21 & 0.61 & 0.65 & 0.025 & 0.028 \\
\hline & Fe- 4kg /fed. & 65.62 & 73.98 & 0.59 & 0.63 & 0.021 & 0.024 \\
\hline & Molasses 200 kg/fed & 69.15 & 76.31 & 0.56 & 0.50 & 0.013 & 0.021 \\
\hline & Molasses 300 kg/fed & 67.59 & 75.11 & 0.58 & 0.56 & 0.019 & 0.023 \\
\hline & Priming at $5 \mathrm{EC} / 24 \mathrm{~h}$ & 60.42 & 71.95 & 0.57 & 0.59 & 0.031 & 0.035 \\
\hline & Priming at $6 \mathrm{EC} / 24 \mathrm{~h}$ & 59.87 & 66.05 & 0.59 & 0.65 & 0.033 & 0.044 \\
\hline \multicolumn{2}{|r|}{ Mean } & 62.68 & 70.10 & 0.60 & 0.62 & 0.029 & 0.034 \\
\hline \multirow{7}{*}{3000 ppm } & Control & 49.60 & 53.72 & 0.76 & 0.85 & 0.105 & 0.115 \\
\hline & Fe- $3 \mathrm{~kg} /$ fed. & 61.12 & 64.37 & 0.67 & 0.69 & 0.033 & 0.038 \\
\hline & Fe- $4 \mathrm{~kg} /$ fed. & 65.32 & 69.50 & 0.66 & 0.68 & 0.025 & 0.028 \\
\hline & Molasses $200 \mathrm{~kg} / \mathrm{fed}$ & 67.72 & 75.62 & 0.64 & 0.63 & 0.019 & 0.024 \\
\hline & Molasses $300 \mathrm{~kg} / \mathrm{fed}$ & 67.27 & 72.97 & 0.65 & 0.65 & 0.024 & 0.026 \\
\hline & Priming at $5 \mathrm{EC} / 24 \mathrm{~h}$ & 59.32 & 65.77 & 0.68 & 0.68 & 0.038 & 0.044 \\
\hline & Priming at $6 \mathrm{EC} / 24 \mathrm{~h}$ & 57.37 & 62.77 & 0.69 & 0.73 & 0.052 & 0.066 \\
\hline \multicolumn{2}{|r|}{ Mean } & 61.10 & 66.39 & 0.68 & 0.70 & 0.042 & 0.049 \\
\hline \multirow{7}{*}{$4000 \mathrm{ppm}$} & Control & 41.65 & 50.36 & 1.08 & 1.10 & 0.127 & 0.127 \\
\hline & Fe- 3kg / fed. & 59.92 & 63.84 & 0.90 & 0.89 & 0.039 & 0.044 \\
\hline & $\mathrm{Fe}-4 \mathrm{~kg} / \mathrm{fed}$ & 64.77 & 65.96 & 0.89 & 0.79 & 0.037 & 0.035 \\
\hline & Molasses $200 \mathrm{~kg} / \mathrm{fed}$ & 65.92 & 70.16 & 0.87 & 0.75 & 0.029 & 0.026 \\
\hline & Molasses $300 \mathrm{~kg} / \mathrm{fed}$ & 65.70 & 68.86 & 0.88 & 0.78 & 0.032 & 0.031 \\
\hline & Priming at $5 \mathrm{EC} / 24 \mathrm{~h}$ & 57.90 & 62.57 & 0.91 & 0.83 & 0.046 & 0.051 \\
\hline & Priming at $6 \mathrm{EC} / 24 \mathrm{~h}$ & 55.77 & 60.56 & 0.93 & 0.87 & 0.065 & 0.067 \\
\hline \multicolumn{2}{|r|}{ Mean } & 58.80 & 63.19 & 0.92 & 0.86 & 0.054 & 0.054 \\
\hline \multirow{7}{*}{$\begin{array}{l}\text { Mean of } \\
\text { Organic and } \\
\text { chemical } \\
\text { compounds }\end{array}$} & Control & 47.75 & 53.40 & 0.85 & 0.90 & 0.098 & 0.103 \\
\hline & Fe- 3kg / fed. & 61.72 & 66.47 & 0.73 & 0.75 & 0.032 & 0.037 \\
\hline & Fe- $4 \mathrm{~kg} / \mathrm{fed}$ & 65.23 & 69.81 & 0.71 & 0.70 & 0.028 & 0.029 \\
\hline & Molasses $200 \mathrm{~kg} / \mathrm{fed}$ & 67.59 & 74.03 & 0.69 & 0.63 & 0.020 & 0.024 \\
\hline & Molasses 300 kg/fed & 66.85 & 72.31 & 0.70 & 0.66 & 0.025 & 0.027 \\
\hline & Priming at $5 \mathrm{EC} / 24 \mathrm{~h}$ & 59.21 & 66.76 & 0.72 & 0.70 & 0.039 & 0.043 \\
\hline & Priming at $6 \mathrm{EC} / 24 \mathrm{~h}$ & 57.67 & 63.13 & 0.73 & 0.75 & 0.050 & 0.059 \\
\hline \multirow{3}{*}{ L.S.D. at $5 \%$} & Salinity & 0.12 & 0.16 & 0.06 & 0.04 & 0.002 & 0.001 \\
\hline & $\begin{array}{c}\text { Organic and Chemical } \\
\text { Compound }\end{array}$ & 0.09 & 0.11 & 0.01 & 0.01 & 0.006 & 0.005 \\
\hline & Interaction & 0.15 & 0.19 & 0.02 & 0.02 & 0.011 & 0.009 \\
\hline
\end{tabular}


treatments. This means that, the highest proline percentage was recorded with tomato plants which were irrigated by water contained the highest salinity levels, i.e. 4000 ppm and no supplied organic or chemical compounds. The opposite was occurred when using the lowest salinity levels, i.e. 2000 ppm as irrigation water and supplying molasses at rate of $200 \mathrm{~kg} / \mathrm{fed}$.

Regarding $\mathrm{Fe}$, data showed that, Fe percentage in leaf tissues significantly decreased with increasing levels of salinity, where the greatest value of Fe percentage was obtained by using saline water at 2000 ppm, while the lowest value was produced by using the highest $\mathrm{NaCl}$ level, i.e. 4000 ppm (Table 5). From another point of view, the effect of mineral and organic compounds on $\mathrm{Fe}$ in tissue of tomato leaves, the obtained data concluded that the application of these compounds resulted in a significant increase in Fe value compared with the control in both two seasons.

Moreover, the maximum increment of Fe content was obtained via using molasses at rate of $200 \mathrm{~kg} / \mathrm{fed}$. compared with other compounds, while the lowest value of $\mathrm{Fe}$ was obtained with the control treatment in both seasons. Concerning to the effect of the interaction between the tested saline levels and the mineral or organic compound treatments on the $\mathrm{Fe}$ of tomato leaf tissues, data in Table (5), indicated that the Fe significantly increased with application the mineral or organic materials, as compared with the control treatments, these were under different salinity levels. As a general, it could be abstracted that, the highest Fe was found with that tomato plants which irrigated with lowest $\mathrm{NaCl}$ level (2000 ppm) and supplied molasses at rate of $200 \mathrm{~kg} / \mathrm{fed}$. On the contrary, the lowest $\mathrm{Fe} \%$ was resulted with that plants which irrigated with water contained the highest $\mathrm{NaCl}$ level (4000 ppm) and no supplied any of organic or mineral compounds.

\section{DISCUSSION}

The aim of this study was to alleviate the harmful effects of saline water irrigation on tomato hybrid Super Strain B by using some chemical and organic compounds such as $\mathrm{Fe}$, priming tomato seedlings roots with $\mathrm{NaCl}$ salt solution and Molasses treatment. Salinity is an environmental stress that limits growth and development in plants. Thus, the irrigation with saline water from 2000, 3000 up to $4000 \mathrm{ppm}$ in this experiment induced changes in the vegetative growth, chemical contents and yield and its components. In this investigation, the irrigation with saline water from 2000 to $4000 \mathrm{ppm}$ caused reduction on plant height, plant fresh and dry weight and leaf area.

The harmful effect of irrigation with saline water over 2000 ppm on plant height, plant fresh and dry weight and leaf area may related to the increase in osmotic pressure of saline root media Mass \& Hoffman, (1977) to alters in the mineral composition in plant which cause ion imbalance or toxicity Greenway \& Munns, (1980), reduction of plant photosynthesis Schwarz et al (2002), reduction of plant stomata conductance Marcelis \& Van Hooijdonk, (1999), the inhibition of both merestimatic activity and elongation of cells Ewais, (1998) and the confusion in metabolic activates that might be affected by the decrease in water absorption and disturbance of mineral balance or both together El- Nimr, (1986).

Regarding dry weight percentage, total chlorophyll content, total $\mathrm{N}, \mathrm{P}, \mathrm{K}$ and $\mathrm{Na}$ under irrigation with saline water from 2000 to $4000 \mathrm{ppm}$, reflected two different trends. The first trend pointed to a positive increase in $\mathrm{Na}$ percentage by increasing saline water levels from 2000, 3000 up to 4000 ppm. Regarding the increase in Na percentage in tomato leaves by increasing saline water irrigation levels from 2000 to $4000 \mathrm{ppm}$ may be attributed to the rise of $\mathrm{pH}$ level in the root zone resulted from salinity led to unavailability of potassium and calcium for the plant and also leads to accumulation of sodium inside the leaves Mahdi and El- Katony, (2001). The second trend showed a negative drop in total chlorophyll content, N, P, K and Fe in tomato leaves with every increase in saline levels beyond the level of $2000 \mathrm{ppm}$. The adverse effect of saline water irrigation on total chlorophyll content in tomato leaves in this study is attributed to the role of salinity in this respect it caused an adverse effect on water relationship of plant consequently decrease photosynthesis process Ahmed, (1998). Also, those harmful effects of salinity attributed to the inhibitory effects on the activity of iron that reflect on reduction in rate of chloroplast structure and chlorophyll accumulation in tomato plants Chougui et al (2004). From another point of view salinity adversely affect the carbon fixation in photosynthesis, the lowest photosynthetic ability under salt stress conditions was due to stomatal closure, inhibition of chlorophyll synthesis or due to decrease in the absorption of minerals needs for chlorophyll biosynthesis i.e. iron Chougui et al (2004) and magnesium Ahmed, (1998). As for the 
harmful effect of saline water irrigation over 2000 ppm on $\mathrm{N}$ percentage in tomato leaves in this investigation might occur by $\mathrm{NO}_{3}^{-} / \mathrm{Cl}^{-}$interaction at the sites for ion transport Cram, (1983) and / or because sodium ions cause severe membrane depolarization in tomato Suhayda et al (1990). In addition, lower $\mathrm{NO}_{3}{ }^{-}$nitrogen is observed in xylem sap of salinized tomato plants because the site of $\mathrm{NO}_{3}{ }^{-}$reduction and assimilation is shifted from shoot to root by salinity Cramer et al (1995). Also, lower stem $\mathrm{K}^{+}$concentration in salinized compared to non- salinized tomato plants suggests reduced functioning of $\mathrm{NO}_{3}{ }^{-}$transport by a $\mathrm{K}^{+}$- shuttle and this could explain the lower nitrate concentration in leaves of salinized plants Cramer et al (1995). Concerning the observed reduction in $\mathrm{P}$ percentage of tomato leaves in our investigation, Pessarakli and Tucker (1988) found that, tomato plants grown with saline water have a significantly lower water uptake by roots, which led to decreased phosphorus uptake and other elements from soil solution to plant. In addition, salinity slows tomato root growth and increases the length of dead roots Snapp and Shennan, (1992) this led to a decrease in phosphorus uptake and other elements from soil solution to plant. In the same trend, Martinez et al (1996) found that, salinity inhibits phosphorus uptake by root, phosphorus translocation from root to shoot and retranslocation of $P$ from old to young leaves perhaps due to decreased mobility of $P$ stored in vacuoles. The decline in potassium uptake in tomato leaves in salinized tomato plants is more than proportional to the reduction in water uptake because interaction between $\mathrm{Na}^{+}$and $\mathrm{K}^{+}$ contributes to decreasing $\mathrm{K}^{+}$uptake Adams and Ho, (1995). Also, the rise of $\mathrm{pH}$ level in the root zone resulted from salinity led to unavailability of potassium for the plant Souheil and Coudret, (1991).

In this study, the data indicated that Fe in tomato leaves decreased by increasing salinity level, this probably due to the high $\mathrm{pH}$ which make $\mathrm{Fe}$ unavailable for the plant Dahiya and Mahendras (1979).

Regarding to the increase in proline content by increasing saline water levels from 2000, 3000 and 4000 ppm is caused by activation of its biosynthesis and deactivation of its degradation. Capability to accumulate proline in response to environmental stress in highly variable between or within species Lutts et al (1996). Proline is synthesized in plants through two alternate pathways: L ornithine and $\mathrm{L}$ - glutamate pathways Parvaiz and Satyawati, (2008). It acts as a signaling molecule initiating adaptation processes towards the stress episode Maggio et al (2002). It provides membrane stability and alleviates the cell membrane disruption brought about by salinity stress Mansour, (1998).

Also in this study the irrigation with saline water levels from 2000, 3000 up to 4000 ppm induced changes in tomato fruits. In our investigation saline water irrigation levels from 2000 to $4000 \mathrm{ppm}$ caused a reduction in some marketable yield and total yield, but it caused an increases in unmarketable yield. The negative effect in marketable and total yield with every increase in saline water levels beyond the level of 2000 ppm attributed to the adverse effect of saline water irrigation up to the level of 2000 ppm on leaf area (Table, 1), total chlorophyll content (Table, 2), NPK percentages in leaves (Table, 3) which may be consequently reduced marketable and total yield. On the other hand, in this study, the increasing in unmarketable yield by saline water irrigation up to $2000 \mathrm{ppm}$ level attributed to increasing blossom- end rot in tomato fruits which came by reason $\mathrm{Ca}^{+2}$ and $\mathrm{K}^{+}$ deficiency in tomato fruit by increasing saline water irrigation Eata, (2001).

In our investigation using the chemical and organic compounds such as $\mathrm{Fe}$, molasses and priming seedlings roots in $\mathrm{NaCl}$ salt solution, under the various salinity levels showed that all these compounds alleviated the effect of salinity and produced the best values compared with the control (without chemical and organic compounds). These compounds proved to be the most effective in producing the longest plant height, the heaviest plant fresh weight, the greatest leaf area, the largest total chlorophyll, the highest concentration of NPK in tomato leaves and increased marketable and total yield.

The question here is how the chemical and organic compounds induce the highest increase in the majority of tomato characteristics under saline water irrigation? The suggested explanations for the significant increase in the majority of studied characteristics in both seasons obtained from using chemical and organic compounds under saline water irrigation. Hence, regarding the favorable effect of molasses on tomato plant growth and productivity under saline water irrigation attributed to molasses contain glycinebetaine material is as a compatible solute in osmotic adjustment of the cytoplasmic compartments where it may accumulate while ions are sequestered in the vacuole Salisbury and Ross, (1992). In addition, glycinebetaine was found to protect protein and 
membrane functions from stress conditions such as drought and salt stresses by playing an antitranspiration agent Hanson et al (1995). Also, application of glycinebetaine increased the net photosynthesis of water stressed tomato plants, this was mostly due to increased stomatal conductance and decreased photorespiration of treated plants Makela et al (1999). Another explanation showed that glycinbetaine has been reported to affect directly the water content of the plants, Seneoka et al (1995) found that, leaf tissue of a maize isoline synthesizing glycinebetaine maintained higher relative water content and turgor when grown in salt stress then an isoline deficient in glycinebetaine synthesis. In addition, Glycinebetaine improved maize plant growth under stress conditions such as drought and salt stresses, these improvements probably resulted from the well-known physiological function of endogenously synthesized glycinebetaine that improves drought tolerance as a cytoplasmic osmoticum, it enables the plant to maintain photosynthetic activity in osmotic stress conditions, stabilize the enzymes involved in amino acid metabolism and maintain turgor pressure even at leaf concentrations Laurie and Stewart, (1990).

The observed enhancement in plant growth and productivity of tomato plants due to primed seedlings roots in $\mathrm{NaCl}$ salt solution may be attributed to tomato roots growth can continue during periods of water stress because effects of water stress are counteracted by osmotic adjustments with Sugars and amino acids such as proline and also with inorganic ions such as nitrate, phosphate, sodium and potassium Taylor et al (1982). Another explanation showed that tomato root cell can modulate the electrostatic properties of the plasma membrane in response to high external salt concentrations and this may have an effect upon salt uptake Suhayda et al (1990).

The promoting effect of Fe on plant growth and productivity of tomato plants under salinity stress may be due to improvement in photosynthesis because of Fe application led to improvement in stomatal conductance Chougui et al (2004). Also, Fe application improved the transfer of $\mathrm{Ca}^{++}$and $\mathrm{K}^{+}$ Chougui et al (2004) as salinity commonly disturbs this transfer and negatively reduce the content of those nutrient in the cells Ashraf and Ashraf, (2003).

\section{CONCLUSION}

Although applied beet molasses, priming tomato seedling in saline water and Fe - EDHHA improved plant performance and production in this study, the effect of salinity was dominant specially with higher levels. However, the application of the previous treatments reduced the negative effects of saline water irrigation, molasses at rate of 200 $\mathrm{kg} / \mathrm{fed}$. came in the first rink in reduced the negative effects of salinity compared to other treatments.

\section{REFERENCES}

Abdel-Mawgoud A.M.R., Stanghellini C., Boehme M., Abou-Hadid A.F. and El-Abd S.O. 2004. Sweet pepper crop responses to greenhouse climate manipulation under saline conditions. Acta Hort., 659, 431-438.

Abdel-Mawgoud A.M.R., El-Nemr M.A., Tantawy A.S. and Habib H.A. 2010. Alleviation of salinity effects on green bean plants using some environmental friendly materials. J. of Applied Sci. Research, 6(7), 871-878.

Adams P. and Ho L.C. 1995. Uptake and distribution of nutrients in relation to tomato fruit quality. Acta Horticulturae, 412, 374-387.

Ahmed Y. 1998. Response of tomato plants to the salinity of irrigation water. Ph.D. Thesis, Fac. of Agric. Zaga. Univ., Benha Branch, 31, 114128.

Ashraf M., Kausar A. and Ashraf M.Y. 2003. Alleviation of salt stress in pearl millet (Pennisetum glaucum) through seed treatments. Agronomie, 23, 227-234.

Bates L.S., Walden R.P. and Tears I.D. 1973. Rapid termination of free proline for water stress studies. Plant Soil 39, 205-208.

Brown J.D. and Lilleland O. 1946. Rapid determination of potassium and sodium in plant material and soil extracts by flamephotometry. Proc. Amer. Society Hort. Sci., 38, 341-364.

Chapman H.D. and Pratt F.P. 1982. Determination of minerals by titration method. Methods of Analysis for Soils, Plants and Water, $2^{\text {nd }}$ Ed. Oakland, CA: Agric. Division, California Univ., USA, pp. 169-170. 
Chougui S., Yaklef N. and Abdel-Hamid D. 2004. Interaction iron - Salinity. 1. Relation of photo synthesis / $\mathrm{K}^{+}$(Roots) with other morphological, physiological, and biochemical aspects of tomato (Lycopersicon esculentium Mill). Arab Univ. J. Agric. Sci., Ain Shams Univ., Cairo, Egypt, 12(2), 481-497.

Cram W.J. 1983. Chloride accumulation as a homeostatic system: set points and perturbation. J. EXP. Bot., 34, 1484 - 1502.

Cramer M.D., Schierholt, A., Wang Y.Z. and Lips S.H. 1995. The influence of salinity on the utilization of root anaplerotic carbon and nitrogen metabolism in tomato seedlings. J. EXP. Bot., 46, 1569 - 1577.

Dahiya S.S. and Mahendras S. 1979. Effect of salinity, alkalinity and iron sources on availability of Iron stress diminishes iron. Plant and Soil, 51, 13-18.

Eata A.E.K. 2001. Response of some tomato cultivars to natural soil salinity and use of some treatments to reduce salt injury. Ph.D. Thesis, Fac. of Agric. Mansoura Univ., Mansoura, Egypt, 40, 1343-1357.

El-Nimr M.H. 1986. Response of some vegetable crops to salinity levels and growth regulators. Ph.D. Thesis, Fac. of Agric. Cairo Univ., Egypt.

Ewais M.A. 1998. Chemical and vital studies on effect of salinity on onion. M.Sc. Thesis, Fac. of Agric. Cairo Univ., Egypt, 37, 1346-1359.

FAO STAT, 2011. www.faostat.org.

Gehad A. 2003. Deteriorated soils in Egypt: Management and Rehabilitation. Executive Authority for Land Improvement Projects (EALIP), Ministry of Agric., Egypt.

Gomaa R.M. 2017. Effect of some Nano - chemical compounds on salinity tolerance for Green Bean Plants. M.Sc. Thesis, Fac. of Agric. Ain Shams Univ., Cairo, Egypt.

Gomez K.A. and Gomez A.A. 1984. Statistical procedures for agriculture research. Second Ed. Wiely Interscience Publ. John Wiley \& Sons, New York, USA, 680 p.

Greenway H. and Munns R. 1980. Mechanism of salt tolerance in non-halophytes. Annual. Rev. Plant Physiol., 31, 149-190.

Hanson A.D., Rivoal J., Burnet M. and Rathinasabapathi B. 1995. Biosynthesis of quaternary ammonium and tertiary sulphonium compounds in response to water stress. In Environment and plant Metabolism. (CAB Abstract 1989-1997). Ed. N. Smirnof. pp. 189-198. Lancaster.
Kacar B. 1972. Bitki ve Topraig n Kimyasal Analizleri II. Bitki Analizleri. Ankara Universitesi Ziraaat Fakültesi Yay Nlar No. 453. Uygulama Klavuzu: 155 p.

Laurie S. and Stewart G.R. 1990. The effect of compatible solutes on the heat stability of glutamine synthetetase from chickpeas grown under different nitrogen and temperature regimes. J. Exptal. Bot., 44, 1415-1422.

Li Y. 2000. Analysis of Greenhouse Tomato production in Relation to Salinity and Shoot Environment. Ph.D. thesis, Wageningen Agricultural University. 95 p.

Lutts S., Kinet J.M. and Bouharmont J. 1996. Effects of salt Stress on growth, mineral nutrition and proline accumulation in relation to osmotic Adjustment in Rice (Oryza sativa L.) cultivars differing in salinity resistance. Plant Growth Reg. 19, 207 - 218.

Maggio A., Miyazaki S., Veronese P., Fujita T., Ibeas J.I., Damsz B., Narasimhan M.L., Hasegawa P.M., Joly R.J. and Bressan R.A. 2002. Does proline accumulation play an active role in stress-induced growth reduction? Plant J. 31, 699-712.

Mahdi M.M. and El-Katony M.T. 2001. Salt tolerance of two wheat genotypes in response of the form of mitroges. Agronomie, 21, 259 - 266.

Makela P., Kontturi M., Pehu E. and Somersalo S. 1999. Photosynthetic response of drought and salt stressed tomato and turnip rape plants to foliar applied glycinebetaine. Physiologia Plantarum, 105, 45-50.

Mansour M.M.F. 1998. Protection of plasma membrane of onion epidermal cells by glycinebetaine and proline against $\mathrm{NaCl}$ stress. Plant Physiol. Biochem., 36, 767-772.

Marcelis L.F. and Van Hooijdonk J. 1999. Effect of salinity on growth, water use and nutrient use in radish (Raphanus sativus). Plant and Soil, 215, 57-64.

Martinez V., Bernstein N. and Lauchli A. 1996. Salt-induced inhibition of phosphorus transport in lettuce plants. Physiol. Plantar., 97, 118-122.

Mass E.V. and Hoffman G.J. 1977. Crop salt tolerance. Amer. Soc. Civil. Eng., IR2; 103-115134.

Munns R. and Tester M. 2008. Mechanisms of salinity tolerance. Annu. Rev. Plant Biol., 59, 651-681.

Parvaiz A. and Satyawati S. 2008. Salt stress and phyto-biochemical responses of plants - a review. Plant Soil Environ., 54, 89 - 99. 
Pessarakli M. and Tucker T. 1988. Dry matter yield and nitrogen -15 uptake by tomatoes under sodium chloride stress. Soil Sci. Soc. Amer. J., 52, 698-700.

Salama Y.A.M. 2003. Adaptation studies on increasing salt tolerance of mung bean plants. M.Sc. Thesis, Fac. Agric Zagazing Univ. (Benha Branch).

Salama Y.A.M., Hassan N.M.K., Saleh S.A. and Zaki M.F. 2012. Zinc amelioration effects on tomato growth and production under saline water irrigation conditions. J. of Applied Sci. Research, 8(12), 5877-5885.

Salisbury F.B. and Ross C.W. 1992. Environmental physiology. In "Plants Physiology", $4^{\text {th }}$ ed. Wadsworth Pup. Comp. USA. pp. 549-600. (CAB Abstr. 1973-1995).

Schwarz D., Klaring H.P., Van lersel M.W. and Ingram K.T. 2002. Growth and photosynthetic response of tomato nutrient solution concentration at two light levels. J. Amer. Soc. Hort. Sci., 127, 984-990.

Seneoka H., Nagasaka C., Hahn D.T., Yang W., Premachandra G.S., Joly R.J. and Rhodes D. 1995. Salt tolerance of glycinebetaine deficient and containing maize lines. Plant Physiol., 107, 631-638.

Shafshak Nadia S., Aid S.M., Khafaga H.S. and Salama Y.A.M. 2008. Improving growth and productivity of tomato under saline conditions by fertilization and salinity hardening. J. Agric. Sci. Mansoura Univ., 33(11), 7803 - 7815.

Snapp S.S. and C. Shennan 1992. Effect of salinity in root growth and death dynamics of tomato (Lycopersicon esculentum). New Phytologis., 121, 71-79.
Souheil H. and Coudret A. 1991. Effect de iadjunction de $\mathrm{KCl}$ de $\mathrm{CaCl}_{2}$ Surla tolerance $\mathrm{NaCl}$ chez deux cultivars triticales. Can. J. Bot., 69: $2113-2121$.

Suhayda C.G., Giannini J.L. and Shannon M.C. 1990. Elestrostatic changes in Lycopersicon esculentum root plasma membrane resulting from salt stress. Plant Physiol., 93, 471 - 478.

Tantawy A.S. 2007. Effect of some mineral and organic compounds on salinity tolerance of tomato. Ph.D. Thesis, Dept. Horticulture, Fac. of Agric., Al-Azhar Univ., Cairo Egypt. 110 p.

Tantawy A.S., Abdel-Mawgoud A.M.R., El-Nemr M.A. and Ghorra Chamoun Y. 2009a. Alleviation of salinity effects on tomato plants by application of amino acids and growth regulators. European J. of Scientific Research, 30(3), 484-494.

Tantawy A.S.A., Helmy Y.I., Abd El-Rehim A.S., Mohamed A.A. and Hewedy A.M. 2009b. Effect of molasses product on salinity tolerance in tomato plants. Egyptian J. of Agric. Sci., 24(8A), 203 - 217.

Tantawy A.S.A., Helmy Y.I., Abd El-Rehim A.S., Mohamed A.A. and Hewedy A.M. 2009c. Effect of root priming on growth and productivity of tomato under salinity stress. Egyptian J. of Agric. Sci., 24(8A), 218-229.

Taylor A.G., Motes J.E. and Kirkham M.B. 1982. Osmotic regulation in germinating tomato seedlings. J. Am. Soc. Horti. Sci., 107, 387-390.

Troug E. and Meyer A.H. 1939. Improvement in deiness colormetic method for phosphorus and aresnic. Ind. Eng. Chem. Anal. Ed., 1, 136139.

Yadava U.L. 1986. A rapid and none destructure method to determine chlorophyll in intact leaves. Hort. Sci., 21, $1449-1450$. 


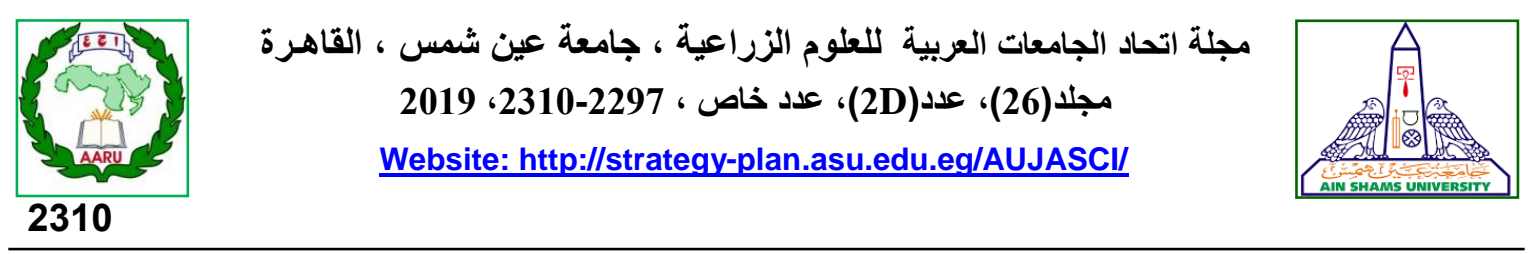

\title{
تأثير مولاس بنجر السكر والحديد المخلبى على نباتات الطماطم تحت ظروف الرى بالماء المالح
}

\author{
[167]

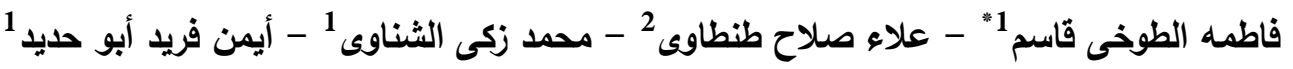

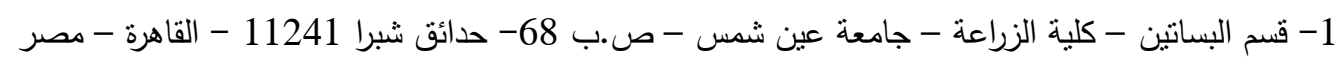

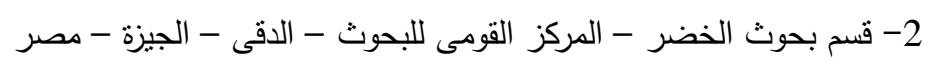

*Corresponding author: fatmaeltoky83@yahoo.com

Received 18 July, 2018

Accepted 19 September, 2018

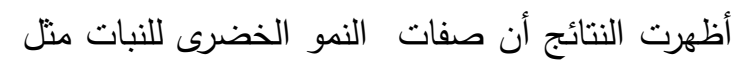

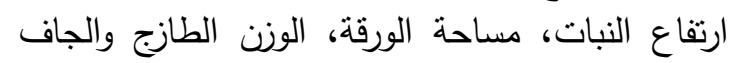
للنبات وكذلك صفات المحصول قد تحسنت بنطبيق

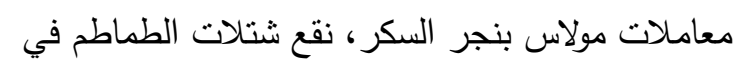

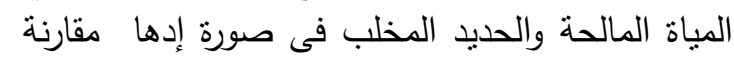
بالنباتات التى لم تعامل (الكنترول) على الترني التيب.

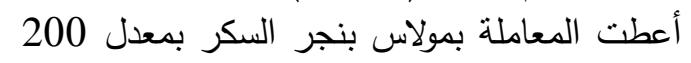

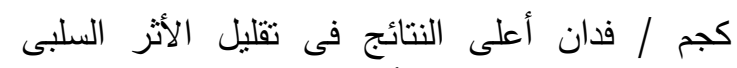

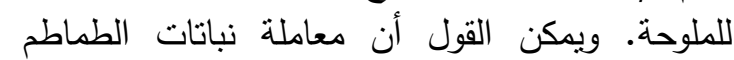
بمولاس بنجر السكر، نقع شتلات الطماطم في المياة

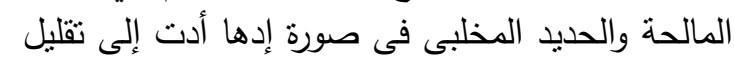

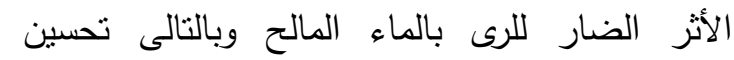

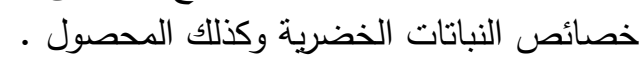

الكلمات الالةة: طماطم، ملوحه، مولاس بنجر السكر ، حديد مخلبى، المحصول

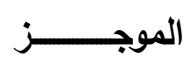

تعد الملوحة عاملاً معيقاً لنمو المحاصيل

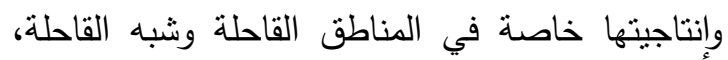

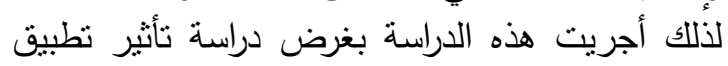

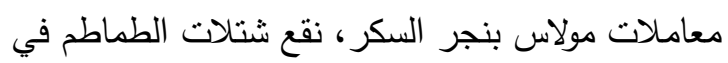

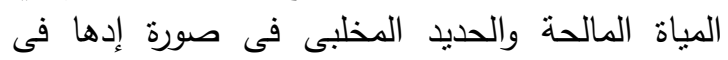
تخفيف الآثار السلبية للملوحة على نباتات الطماطم

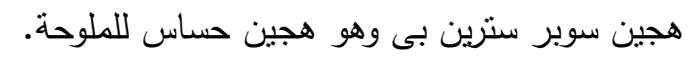
تمت زراعة شتنلات الطماطم الهجين في 4 فئن أبريل

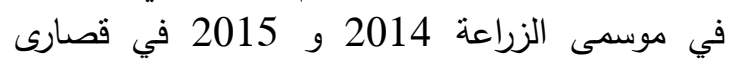

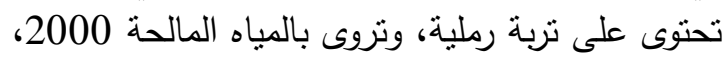

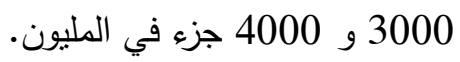
نم إضافة مولاس بنجر السكر بمعدل 200 و و 3000

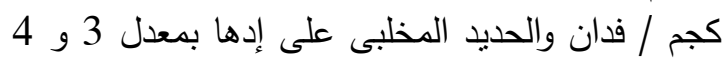

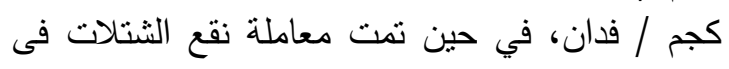
مياه مالحه 5 ، 6 ديسيسيمنز / متر لمدة 24 ساعة. 